\title{
Nanoscale Building Material Characterization by Means of High-Resolution SEM Imaging Techniques
}

\author{
B. E. Möser
}

F. A. Finger-Institute for Building Materials Science, Bauhaus-University Weimar, Germany

Two types of microscopes namely a FEI XL-30 ESEM-FEG and a FEI Nova NanoSEM 230, were used. These devices are capable of different high-resolution imaging and analytical techniques (EDS, EBSD) in order to characterize water containing and charging building materials. In this field the environmental-SEM (ESEM) technology in connection with high-resolution electron microscopy is especially important, because water vapor can be used as gas in the sample chamber. The ESEMFEG can operate under a high water vapor pressure up to 13.3 mbar. By means of a Peltier stage the in-situ characterization of dynamic processes such as crystallization, dissolution, hydration (e. g. calcium sulfate hemihydrates) as well as a quasi continuous screening of the microstructural development of fresh cement pastes etc. become possible. Comparative studies between conventional high vacuum and ESEM conditions show that the water containing microstructures and phases are changed in their morphologies and their chemical compositions. This is caused by loss of capillary, adsorptive and chemically bound water under high vacuum conditions. The above mentioned process even leads in some cases to the destruction of the lattice structure. The mineral ettringite e. g. has 32 molecules chemically bound water and loses approximately 20 molecules in high vacuum.

The Nova NanoSEM 230 combines magnetic immersion lens technology with ESEM technology. As shown in Fig.1 the combined effect and the usage of a Helix gaseous secondary electron detector [1] yields unique high resolution low vacuum characterization capabilities of insulating samples in a water vapor environment under low voltage conditions (smaller than $5 \mathrm{kV}$ ). Low energy electrons reduce beam penetration and optimize the amount of surface information carried by the signal. Only by deploying very low accelerating voltages of smaller than $1.5 \mathrm{kV}$ it becomes possible to distinguish between the $\mathrm{C}-\mathrm{S}-\mathrm{H}$ phases and the pore solution in the microstructure of cementitious materials.

This microscope can also be used as a cryo-SEM for the investigation of aqueous samples in high vacuum environment. The high pressure freezing technique used in this context has been adapted from life sciences [2] to satisfy the needs for building material research. The cryo-SEM, the low vacuum SEM (up to 1.6 mbar) as well as the ESEM (up to 13.3 mbar) methods have their own merits and are helpful when they are used together. High resolution, low-voltage SEM (LV-SEM) is also a powerful tool for studying very dense and compact microstructures of ultra-high performance concrete (UHPC [3], Fig. 2) as well as the nano-scale phases in it. Another aspect is the visualization of nano-sized reactive materials such as silica fume (as shown in Fig. 3), aerosol, nanogel, carbon nanotubes, metakaolin (as shown in Fig. 4), C-S-H-seeds and their reaction behavior in the cementitious matrix.

Because LV-SEM provides images having a clear topographic contrast even on specimens containing phases with low density, such as hydrates, the confusion of structural overlap does not occur. Furthermore, in microanalytical measurements the application of the LV-SEM mode reduces the excitation volume of the electrons up to a range of smaller than $500 \mathrm{~nm}$ and achieves a better Xray emission for light elements. 


\section{References}

[1] B.L. Thiel et al., Microsc. Microanal. 10 (Suppl. 2) (2004) 1058.

[2] H. Schatten et al., Biological low-Voltage Scanning Electron Microscopy, Springer Science+Business Media, LLC, New York, 2008

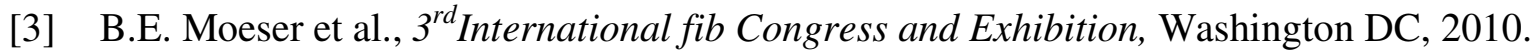

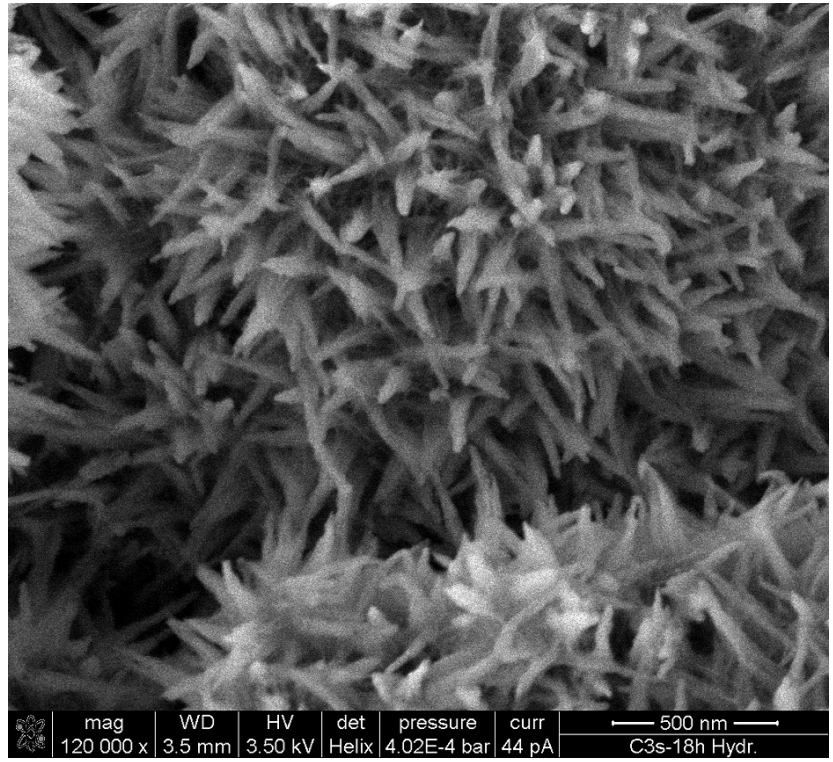

FIG. 1. Artefact-free low voltage, low vacuum imaging of C-S-H phases - the hydration product of $\mathrm{C}_{3} \mathrm{~S}$ - by means of a Helix detector.

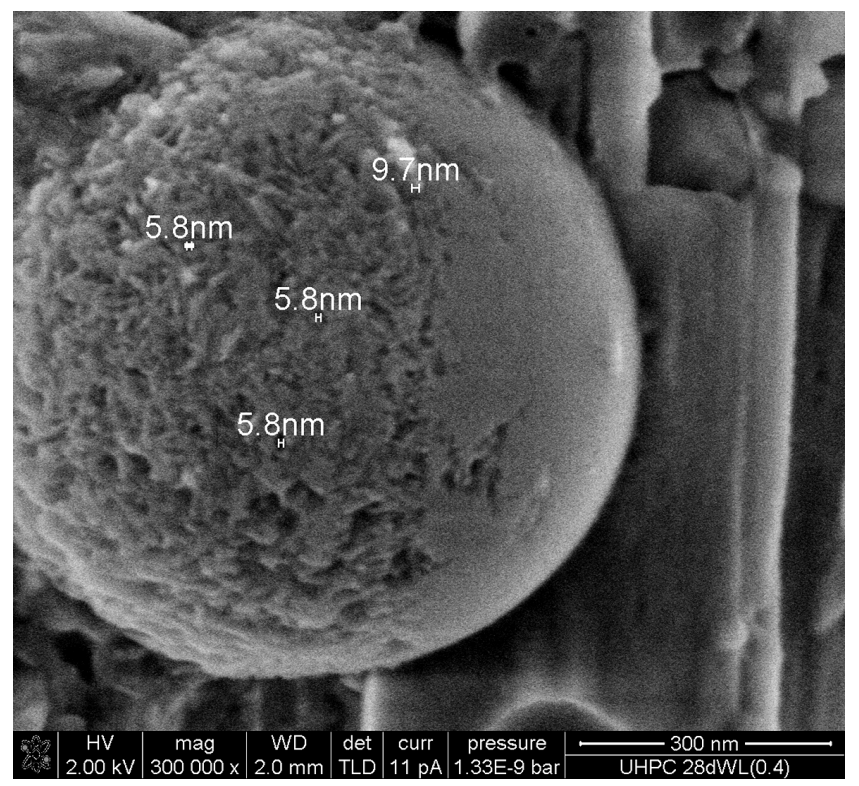

FIG. 3. Direct comparison of a pozzolanic reaction in progress: (left) silica fume particle with signs of dissolution and right embedded in portlandite aggregate in its original state.

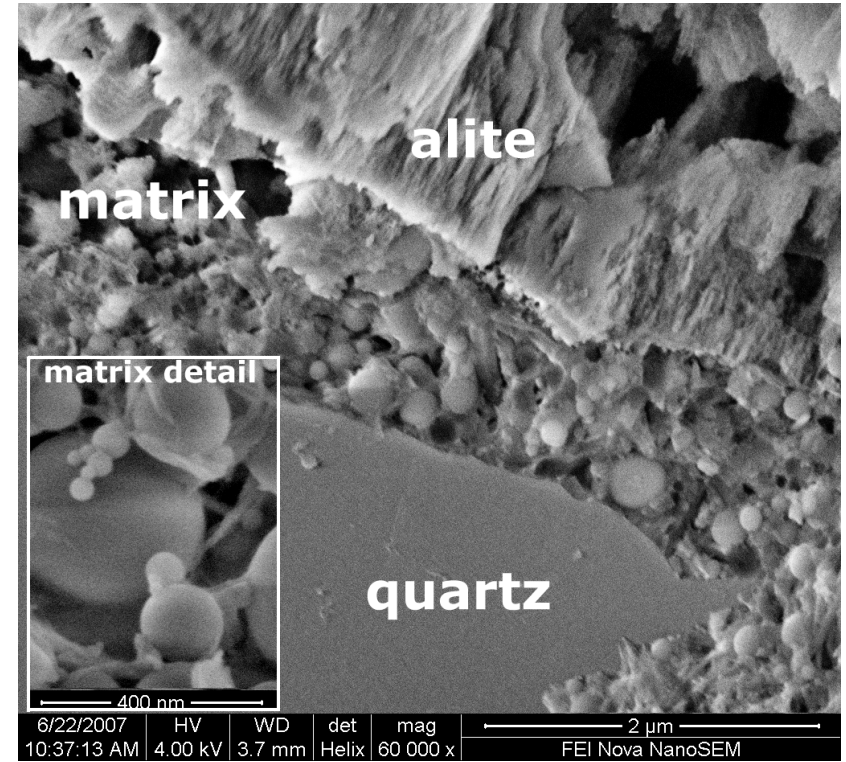

FIG. 2. UHPC microstructure after $2 d$ hydration time; low voltage, low vacuum imaging using a Helix detector, notice left: matrix enlargement.

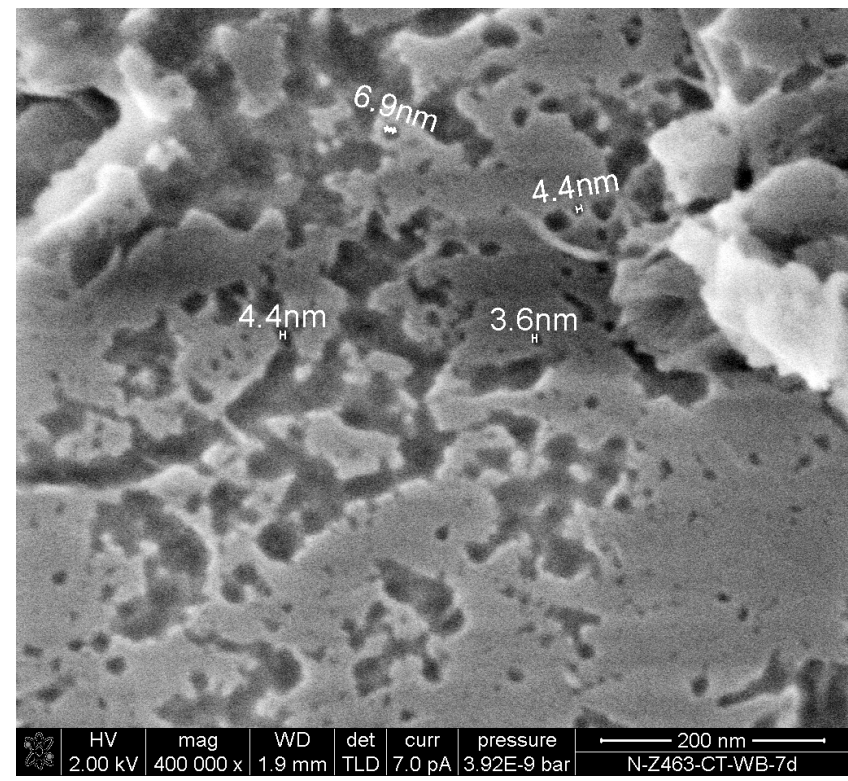

FIG. 4. Dissolution structure on the surface of highly reactive metakaolin due to the pozzolanic reaction in OPC after 7 days hydration time. 